\title{
FACTORES DE RIESGO DE DESERCIÓN PRESENTES EN ALUMNOS REPITENTES DE LAS CARRERAS DE ENFERMERÍA Y KINESIOLOGÍA EN UNA UNIVERSIDAD CHILENA
}

\section{DROPOUT RISK FACTORS IDENTIFIED IN STUDENTS REPEATING COURSES IN NURSING AND KINESIOLOGY PROGRAMS AT A CHILEAN UNIVERSITY}

\author{
Rubén Celis Schneider* \\ Carmen Luz Flores Ramírez ${ }^{* *}$ \\ María Cristina Reyes Martínez ${ }^{* * *}$ \\ Héctor Venegas Villanueva ${ }^{* * *}$
}

\begin{abstract}
RESUMEN
Las altas tasas de repitencia y deserción en alumnos universitarios ha sido tema de preocupación de docentes y autoridades. Objetivo: Describir cómo se presentan los factores de riesgo de deserción en alumnos repitentes de primer año de las carreras de Enfermería y Kinesiología de una universidad chilena. Material y método: Estudio cuantitativo descriptivo en el cual se aplicó un instrumento validado a 83 alumnos que se encontraban matriculados en las carreras señaladas, teniendo como criterio de inclusión que hayan reprobado al menos una asignatura. Se realizó análisis estadístico (SPSS) de la información recopilada. Resultados: se observa que la mayoría de los estudiantes usa como técnica de estudio la lectura de los contenidos seguido de aquellos que subrayan la materia; existe un porcentaje importante de estudiantes que señalan haber cursado una carrera universitaria previa; el mayor número de estudiantes financia sus estudios a través de créditos y becas; el porcentaje más alto pertenece a un grupo con un ingreso económico familiar de hasta $\$ 350.000$ pesos; un gran número de estudiantes cursó su enseñanza media en un establecimiento municipalizado; la gran mayoría de los padres de los estudiantes alcanzó un nivel educacional secundario y secundario incompleto; y finalmente un gran número de estudiantes señala que son los primeros del grupo familiar en ingresar a cursar estudios superiores. Conclusiones: Es posible inferir que en este grupo de estudiantes se encuentra presente algunos de los factores de riesgo de deserción estudiados, lo que los puede llevar a desertar en algún momento de su carrera.
\end{abstract}

Palabras clave: Educación superior, abandono de los estudiantes, factores de riesgo, estudiantes de enfermería, estudiantes de kinesiología.

\begin{abstract}
High repetition and dropout rates for college students have raised concern among teaching personnel and authorities. This paper presents research conducted among first-year Nursing and Kinesiology students at a Chilean university, with the aim of describing how attrition risk factors affect this group of students repeating grades. It is a quantitative descriptive study in which a validated questionnaire was applied to 83 students who were enrolled in these programs, with the selection criterion of having failed at least one course. A statistical

\footnotetext{
*Psicólogo. INACAP, Osorno - Chile. E-mail: r.celis.sch@gmail.com

** Kinesióloga. Clínica Alemana Osorno- Chile. E-mail: carmenluzflores2@gmail.com

${ }^{* * *}$ Enfermera Docente Universidad Mayor Temuco Chile. Av. Alemania 0281 Temuco. E-mail: maria.reyesm@umayor.cl

${ }^{* * * *}$ Asistente Social, Universidad Católica y Universidad Mayor. Temuco- Chile. E-mail: hvenegas@uct.cl
} 
analysis (SPSS) was then applied to the information collected. Results: it was found that the majority of the students surveyed adopt a study technique consisting of reading and then underlining the course materials; a significant percentage indicated that they had previously been enrolled in a different university course; more students finance their studies using credits or scholarships than through any other funding mechanism; the most heavily represented family income group is the group with incomes of up to CLP350,000; a large number of those surveyed received secondary education at a municipally funded school; the vast majority of students' parents had at least started secondary education but never entered the tertiary level; and finally, a large number of students indicated that they are the first in their immediate family to enter any form of higher education. Conclusions: It can be concluded that this group of students presents a number of the dropout risk factors already studied, which could lead them to abandon higher education at any point.

Key words: Education, higher, students dropouts, risk factors, students, nursing, kinesiology.

Fecha recepción: 05/12/11 Fecha aceptación: 04/11/13

\section{INTRODUCCIÓN}

En los últimos 30 años se han producido cambios fundamentales en las normas que regulan la educación superior en nuestro país, lo que junto a un fortalecimiento de la economía y a una creciente necesidad de educación superior, ha permitido que las instituciones que ofrecen formación técnica superior y universitaria hayan sufrido un crecimiento exponencial. Lo anterior ha llevado a que aumente también la demanda de estudios superiores por parte de los jóvenes que egresan de la educación secundaria.

Sin embargo, la eficacia de la educación terciaria es bastante baja, la titulación promedio para los años 1998 - 2002 es de un $43 \%$, siendo el $59 \%$ para las universidades públicas y $37 \%$ para las universidades privadas; el aumento en la deserción universitaria comienza desde hace cuatro décadas, dándose un incremento significativo en la década de 1990. Este problema no es particular de Chile, sino que se observa en el contexto mundial. Esto conlleva además un costo económico para los estudiantes y sus familias, las instituciones y los países, se estima en US\$54 millones en las universidades públicas y en US $\$ 45$ millones para las universidades privadas (1-7).

$\mathrm{Al}$ abandonar la educación superior los estudiantes disminuyen sus aspiraciones de movilidad social y las expectativas que sus padres han depositado en ellos, lo cual suele provocar sentimientos de fracaso afectando su autoconfianza $(5,8,9)$.

La cobertura bruta de la Educación Superior en el país alcanza a un $32 \%$ de la población entre los 18 a 24 años, de los cuales el $75,5 \%$ está matriculado en universidades, $55,4 \%$ matriculado en las 25 universidades adscritas al Consejo de Rectores, y 44,6\% en universidades privadas. En Latinoamérica se observa un $22 \%$ de deserción en universitarios, mientras que en nuestro país esta tasa alcanza a un 19\% en las universidades adscritas al Consejo de Rectores de Universidades Chilenas (CRUCH) y a un $22 \%$ en las universidades privadas autónomas (10).

Todo niño y joven es educable $(9,11)$ va a depender del resultado de la interacción entre factores del hogar y de la escuela. Dentro de los factores que modelan la educabilidad se encuentran: 1) el efecto "vecindario", que se refiere al impacto del entorno próximo; 2) factor "confianza", entendiendo por confianza las expectativas que ponen las personas sobre el comportamiento de los otros; 3 ) la "subjetividad", entendida como los significados particulares que las personas le atribuyen a las bases como la familia, el trabajo y la comunidad. La subjetividad es central en la interacción profesor-alumno, porque el 
significado y representación de los docentes determinan sus atribuciones de causa de éxito o fracaso académico en los estudiantes, basados en sus propios pronósticos de cantidad y calidad de las aptitudes cognitivas de los alumnos; y por último, 4) efecto "políticas sociales", considerando que la educabilidad es un constructo social afectada por las intervenciones sociales (11).

Dentro de los factores explicativos de la deserción se encuentran los sicológicos, centrados en la personalidad del estudiante y se señala que las intenciones de un individuo son el resultado de sus creencias que influyen sobre sus actitudes y comportamiento; de este modo la decisión de desertar o no de los estudios está influida por las conductas previas, actitudes sobre la deserción y/o persistencia, y por normas subjetivas que generan una intención conductual que es un comportamiento definido (9). Se establece que el rendimiento académico previo influye significativamente en el desempeño futuro del estudiante, al actuar sobre su autoconcepto, percepción de la dificultad de los estudios, sus metas, valores y expectativas de éxito (5, 7).

Como causales de deserción, también la literatura muestra factores sociológicos, y se sugiere que la deserción es el resultado de la falta de integración de los estudiantes en el entorno de la educación superior, siendo el medio familiar una de las principales fuentes que expone a los estudiantes a influencias, expectativas y demandas que afectan su nivel de integración social en la universidad (7).

Otros factores que influyen en la decisión de desertar o no son los económicos, organizacionales o institucionales, enfocados a las características de la institución universitaria respecto a servicios y beneficios que ofrece a los estudiantes, teniendo un rol crucial las variables de calidad "de la docencia" y "de las experiencias de los estudiantes en el aula" (1$5,7)$.

Por último figuran como factores de riesgo de deserción los de interacción, el proceso de enseñanza y los docentes cumplen un rol fundamental en la determinación de la deserción de los alumnos. El apoyo de pares cumple un efecto sobre la integración social, y esta última afecta la satisfacción del estudiante, mejorando su inserción en la institución y contribuyendo a reafirmar su compromiso con ésta (7).

Estudios realizados en nuestro país (1- 6) coinciden respecto a la multicausalidad en el fenómeno de la deserción. Dentro de los factores más significativos mencionan la falta de adaptación de las instituciones al nuevo tipo de estudiantes que ingresa al sistema, la desinformación de los estudiantes al momento de optar por una carrera, la extensa duración de éstas, razones vocacionales, motivacionales, económicas, o una combinación de varias de estas variables (7).

Por otra parte también destaca la interacción de factores individuales, sociales e institucionales como causantes de la deserción universitaria. Entre las causas sociales e individuales, Himmel (7) señala los valores, las expectativas personales y familiares, el autoconcepto académico de los alumnos y la familia, entre los más importantes. Dentro de los factores institucionales menciona la falta de integración de los alumnos a su institución, la calidad de la docencia y los sistemas de apoyo institucional.

Para muchos jóvenes, especialmente aquellos que no representan el perfil tradicional de alumno universitario, resulta muy complejo el ajuste a la vida universitaria. Según lo señalado por los propios estudiantes, el principal problema que debieron enfrentar al ingresar a la universidad fue adaptarse al ritmo universitario y desarrollar hábitos de estudio. La evidencia muestra que en muchos casos los jóvenes no están preparados para enfrentar las exigencias universitarias, lo que es manifestado principalmente por alumnos provenientes de colegios particulares subvencionados y municipales. En relación a la deserción universitaria permanente, los factores no académicos resultan deter- 
minantes, siendo las principales causas las económicas y familiares. Las circunstancias como la pérdida del empleo de un familiar directo o que el alumno debe asumir responsabilidades como jefe de familia, constituyen otros tipos de factores centrales $(3,11)$.

Cabe preguntarse por las razones que expliquen esta alta tasa de abandono, siendo que las ofertas son tan variadas y la demanda es tan creciente. A pesar de que no hay estudios que describan la deserción de los estudiantes en la educación superior en Chile, sí es factible encontrar que este fenómeno tendría causas que van más allá de los factores económicos o de motivación, ya que no sólo involucra otros elementos que dependen del individuo, sino que también de la institución misma y de la sociedad. Lo que sí queda claro, es que el dejar los estudios inconclusos conlleva inconvenientes y costos para el estudiante y su familia, para la institución, y también para el Estado, por lo que es un tema del cual deberíamos preocuparnos.

La investigación que se presenta a continuación tiene como objetivo describir la presencia de factores de riesgo de deserción en alumnos repitentes, específicamente en los estudiantes que cursan primer año de las carreras de Enfermería y Kinesiología de una universidad chilena.

\section{MATERIAL Y MÉTODO}

Este estudio es cuantitativo-descriptivo, transversal. En este estudio se utilizó un cuestionario estructurado autoadministrado que se aplicó a todos los alumnos repitentes de las cohortes 2007 y 2008 matriculados a marzo de 2009 en las carreras de Enfermería y Kinesiología de la universidad estudiada. Se elaboraron preguntas cerradas de alternativas de respuestas delimitadas, de tipo dicotómicas, de selección múltiple y escala de Lickert. Las preguntas apuntaron a recoger información de cada uno de los factores de riesgo de deserción universitaria que señala la literatura, factores psicológicos como motivación, autoestima y rendimiento previo; factores económicos como forma de financiamiento de sus estudios, ingreso familiar y ocupación padres; factores sociológicos como establecimiento educacional de origen, escolaridad de los padres; factores institucionales como beneficios que ofrece la institución y son utilizados por los estudiantes; y finalmente factores de interacción como interacción con los docentes en cuanto a calidad y oportunidad.

Dada la población no se trabajó con muestra, sino que se realizó un censo. El criterio de inclusión fue todos los alumnos repitentes de las carreras de Enfermería y Kinesiología de las promociones de 2007 y 2008 matriculados a marzo del año 2009.

\section{Recolección de datos}

Una vez elaborado el cuestionario, dos jueces externos expertos en metodología de la investigación establecieron su validez facial. Posteriormente se aplicó de manera piloto a doce alumnos repitentes de las mismas carreras, pero de otro nivel, que no participaron en esta investigación.

En la base de datos de la universidad se logró identificar a 126 alumnos repitentes de las carreras en estudio, el equipo investigador aplicó el cuestionario validado, logrando reclutar a 83 alumnos de la población definida, que correspondía a estudiantes matriculados a marzo de 2009, y que en forma voluntaria decidieron participar del estudio; de los 83 estudiantes 42 pertenecían a la carrera de Kinesiología y 41 a Enfermería. El cuestionario fue aplicado en un mismo momento a los 83 estudiantes con un tiempo de aplicación promedio de 15 min por cuestionario.

Para el análisis de datos de las variables cuantitativas se utilizó la estadística descriptiva, a través del software SPSS Statistics 15.0. En una primera etapa se describen los datos, 
sus valores o las puntuaciones logradas para cada variable a través de gráficos y tablas. Luego se realizó un análisis descriptivo de cada una de las tablas y gráficos.

Aspectos éticos: se solicitó a la directora de Docencia de la universidad y a los directores de las carreras en estudio autorización para la aplicación del instrumento de recolección de datos a través de una carta formal. Previo a la aplicación de la encuesta se le informó a cada estudiante el objetivo del estudio y se le solicitó su consentimiento informado, dando su permiso para utilizar la información recolectada resguardando el principio de confiabilidad.

\section{RESULTADOS}

Del total de 83 alumnos encuestados 42 cursan la carrera de Kinesiología, siendo 19 del sexo masculino y 23 del femenino, y de los 41 alumnos de la carrera de Enfermería, 10 son de sexo masculino y 31 del femenino.

Dentro de los resultados destaca que un alto porcentaje (31\%) tiene una mala técnica de estudio, sólo lee la materia. Existe un por- centaje no despreciable (22\%) de estudiantes que señala haber cursado una carrera previa. Un $40 \%$ financia sus estudios a través de crédito y le sigue con un $37 \%$ financiamiento a través de los padres. El 46\% de los estudiantes pertenece a los dos grupos de ingresos familiares más bajos, $\$ 350.000$ o menos. En cuanto a la situación laboral de los padres, el $21 \%$ de los padres de los encuestados no tiene un trabajo estable o se encuentra cesante; y el 55\% de las madres no tiene un trabajo remunerado o lo realizan en forma esporádica. En cuanto al establecimiento educacional de origen, el 59\% de los encuestados cursó su enseñanza media en un establecimiento municipalizado. Además destaca, en relación al nivel educacional de los padres, que el padre ha alcanzado sólo en un 36\% educación secundaria completa y sólo un 10\% logró un nivel universitario completo. En cuanto al nivel educacional alcanzado por la madre del estudiante el $41 \%$ de ellas ha completado la educación secundaria y un $9 \%$ logró completar la formación universitaria. Finalmente el 39\% de los encuestados señala ser el primer miembro del grupo familiar que cursa estudios superiores (Tabla 1 ).

Tabla 1. Factores de riesgo de deserción universitaria en alumnos repitentes de primer año de las carreras de Enfermería y Kinesiología de una universidad chilena.

\begin{tabular}{|llr|}
\hline Variable & & Porcentaje \\
\hline Técnica estudio & Lee materia & $31 \%$ \\
& Subrayado & $23 \%$ \\
& Resúmenes y/o mapas conceptuales & $22 \%$ \\
& Memorización & $14 \%$ \\
& Otros & $10 \%$ \\
\hline Repite enseñanza & $\mathrm{Si}$ & $17 \%$ \\
& $\mathrm{No}$ & $83 \%$ \\
\hline Estudios superiores previos & $\mathrm{Si}$ & $22 \%$ \\
& $\mathrm{No}$ & $78 \%$ \\
\hline
\end{tabular}


Continuación Tabla 1.

\begin{tabular}{|c|c|c|}
\hline \multirow[t]{4}{*}{ Forma financiamiento estudios } & Padres & $37 \%$ \\
\hline & Crédito & $40 \%$ \\
\hline & Becas & $13 \%$ \\
\hline & Autofinanciado & $10 \%$ \\
\hline \multirow[t]{5}{*}{ Ingreso grupo familiar $^{*}$} & Hasta $\$ 200.000$ & $26 \%$ \\
\hline & $\$ 201.000-\$ 350.000$ & $20 \%$ \\
\hline & $\$ 351.000-\$ 500.0000$ & $21 \%$ \\
\hline & $\$ 500.001-\$ 999.999$ & $25 \%$ \\
\hline & $\$ 1.000 .000$ o más & $8 \%$ \\
\hline \multirow[t]{3}{*}{ Trabajo padre } & Estable & $79 \%$ \\
\hline & Ocasional & $9 \%$ \\
\hline & Sin trabajo & $12 \%$ \\
\hline \multirow[t]{3}{*}{ Trabajo madre } & Estable & $45 \%$ \\
\hline & Ocasional & $10 \%$ \\
\hline & Sin trabajo & $45 \%$ \\
\hline \multirow[t]{3}{*}{ Establecimiento educacional origen } & Municipalizado & $59 \%$ \\
\hline & Particular subvencionado & $29 \%$ \\
\hline & Particular & $12 \%$ \\
\hline \multirow[t]{8}{*}{ Nivel educacional padre } & Primaria incompleta & $7 \%$ \\
\hline & Primaria completa & $5 \%$ \\
\hline & Secundaria incompleta & $18 \%$ \\
\hline & Secundaria completa & $36 \%$ \\
\hline & Téc. superior incompleto & $6 \%$ \\
\hline & Téc. superior completo & $16 \%$ \\
\hline & Universitario incompleto & $2 \%$ \\
\hline & Universitario completo & $10 \%$ \\
\hline \multirow[t]{8}{*}{ Nivel educacional madre } & Primaria incompleta & $6 \%$ \\
\hline & Primaria completa & $8 \%$ \\
\hline & Secundaria incompleta & $11 \%$ \\
\hline & Secundaria completa & $41 \%$ \\
\hline & Téc. superior incompleto & $4 \%$ \\
\hline & Téc. superior completo & $17 \%$ \\
\hline & Universitario incompleto & $4 \%$ \\
\hline & Universitario completo & $9 \%$ \\
\hline \multirow{4}{*}{$\begin{array}{l}\mathrm{N}^{\circ} \text { integrantes grupo familiar que han } \\
\text { cursado estudios superiores }\end{array}$} & 0 & $39 \%$ \\
\hline & 1 & $27 \%$ \\
\hline & 2 & $18 \%$ \\
\hline & 3 o más & $16 \%$ \\
\hline
\end{tabular}

*Ingreso en pesos chilenos

1 USD $=500$ pesos chilenos 


\section{DISCUSIÓN Y CONCLUSIÓN}

De los resultados obtenidos podemos señalar que hay concordancia con lo expuesto por la literatura, en donde se señala que la situación financiera del estudiante y su familia es un factor de riesgo para su desempeño académico $(3,5)$. En el presente estudio, un 37 $\%$ de los alumnos financian sus estudios a través de sus padres, sin embargo la mayoría cuenta con ingresos económicos inferiores a $\$ 350.000$, por lo que existe un riesgo que los estudios puedan verse interrumpidos debido a la dificultad de poder financiar la carrera. Más aún, al repetir una asignatura y alargar así el período de formación, la familia se encuentra ante la exigencia de continuar apoyando económicamente a su integrante y seguir invirtiendo recursos monetarios por un lapso mayor al planificado inicialmente. Por otra parte, las carreras universitarias cuentan con planes de estudios que implican que si un estudiante reprueba una asignatura, aunque ésta sea de carácter semestral, no cuenta con la posibilidad de retomar esa materia al semestre siguiente, sino que deberá esperar hasta el año próximo para cursarla (2). Esto coincide con dos investigaciones realizadas en estudiantes de enfermería de una universidad de Colombia y una universidad peruana, en donde se muestra que el factor económico fue el que obtuvo gran importancia al tomar la decisión de abandonar los estudios $(12,13)$.

En el estudio de Canales y de los Ríos (3), se menciona que el rendimiento académico de los alumnos está muy vinculado a factores socioculturales. El principal problema que deben enfrentar los jóvenes al ingresar a la universidad tiene que ver con la adaptación al ritmo universitario y a la metodología de estudio. Las dificultades para enfrentar las exigencias universitarias se manifiesta principalmente en alumnos egresados de establecimientos municipalizados; este planteamiento tiene estrecha relación con lo observado en la presente investigación, en que los encuestados provienen en su mayoría de establecimientos municipalizados y en que la técnica de estudio utilizada por casi un tercio de los respondientes se limita a la lectura de textos, sin mayor análisis y sin utilizar otras técnicas con mejores resultados.

La decisión de abandonar o permanecer en sus estudios está afectada por las conductas previas, las actitudes acerca de la retención y deserción, y por la visión que tiene el sujeto acerca de dejar sus estudios, lo que podrá determinar que la persona considere este acto como una alternativa posible (9). Es por eso que la proporción de estudiantes del presente estudio que han cursado y abandonado alguna carrera anteriormente, los constituye en un grupo de riesgo de deserción.

Otro de los factores relacionado con la deserción tiene que ver con las características propias de la familia del estudiante, siendo muy significativo la ocupación y el nivel educacional de los padres (6). El grupo estudiado se caracteriza por provenir de familias donde los padres cuentan con poca preparación académica, donde sólo el 10\% de los padres y el $9 \%$ de las madres cuenta con estudios superiores completos, complementado además con que el mayor número de estudiantes encuestados señala ser el primer miembro del grupo familiar que cursa estudios universitarios, lo que se apoya con los datos del Censo 2002, que señala que la mayoría de los alumnos que ingresan a la educación superior corresponde a la primera generación universitaria en su familia (6, 10).

De acuerdo a los resultados obtenidos, es posible señalar que en este grupo de estudio se encuentran presentes varios de los factores de riesgo de deserción, lo que los hace un grupo especialmente vulnerable. Dentro de estos factores destacan la situación económica del grupo familiar, siendo relevante al momento de tomar la decisión de desertar de los estudios universitarios, junto con el nivel educacional de los padres y conduc- 
tas previas del grupo estudiado (abandono o deserción previos). Por todo esto se concluye que en este grupo de estudio existiría una alta probabilidad de desertar pues se encuentran presentes muchos de los factores de riesgo señalados en la literatura.

Para evitar este problema se hace necesario implementar algunas medidas, entre ellas: programas académicos de apoyo, programas de apoyo social y económico, de motivación e integración, estrategias de retroalimentación temprana, flexibilidad curricular y considerar variables emocionales y cognitivas.

\section{REFERENCIAS}

1. Bernasconi A, Rojas F. Informe sobre la Educación Superior en Chile: 1980- 2003 [Internet]. Santiago, Chile: Organización para las Naciones Unidas para la Educación, la Ciencia y la Cultura (UNESCO); 2004 [citado 25 jul 2008]. Disponible en: http://www.unesco.org/new/es/general-information/about-us/

2. Brunner J. Informe sobre la educación superior en Chile. [Internet] Santiago, Chile: Facultad Latinoamericana de Ciencias Sociales (FLACSO); 1986 [citado 25 julio 2008]. Disponible en http:// cronopio.flacso.cl/fondo/pub/publicos/1984/DT/001008.pdf

3. Canales A, De los Ríos D. Factores explicativos de la deserción universitaria. Revista Calidad en la Educación [Internet] 2007 [citado 27 julio 2008]; 26: 173-201. Disponible en: http://www.cned.cl/public/secciones/SeccionRevistaCalidad/ doc/56/cse_articulo605.pdf

4. Donoso S, Schiefelbein E. Análisis de los modelos explicativos de retención de estudiantes en la universidad: una visión desde la desigualdad social. Estud. pedagóg. [Internet] 2007 [citado 27 jul 2008], 31(1): 7-27. Disponible en http://www. scielo.cl
5. González L, Uribe D, González S. Estudios sobre la repitencia y deserción de la educación superior chilena. [Internet] Santiago, Chile: UNESCO; 2005 [citado 27 jul 2008]. Disponible en: http:// unesdoc.unesco.org

6. Centro Universitario del Desarrollo (CINDA). Repitencia y Deserción Universitaria en América Latina [Internet]. Santiago, Chile: UNESCO; 2006 [citado 28 jul 2008]. Disponible en: http://www. cinda.cl/htm/p0506.htm

7. Himmel E. Modelos de Análisis de la Deserción Estudiantil en la Educación Superior. Revista Calidad en la Educación [Internet] 2002 [citado 30 jul 2008]; 17(2): 91-108. Disponible en: http://www.cned. $\mathrm{cl} /$ public/secciones/SeccionRevistaCalidad/doc/35/cse_articulo141.pdf

8. López N, Tedesco JC. Las condiciones de educabilidad de los niños y adolescentes en América Latina. [Internet]. Buenos Aires: Instituto Internacional de Planeamiento de la Educación; 2002 jun [citado 12 oct 2008]. Disponible en: http://www. iipe-buenosaires.org.ar/node/79

9. Navarro L. La escuela y las condiciones sociales para aprender y enseñar: equidad social y educación en sectores de pobreza urbana. [Internet] Buenos Aires: UNESCO; 2004 [acceso 30 julio 2008]. Disponible en http://www.oei. es/reformaseducativas/educacion_ reforma_equidad_chile_iipe.pdf

10. MINEDUC.cl. Compendio Estadístico [Internet] Chile: Mineduc.cl 2007 [citado 12 oct 2008]. Disponible en http:// compendio.educasup.cl/

11. Nef J. El Concepto de Estado Subsidiario y la educación como un bien de mercado: un bosquejo de análisis político. Enfoques Educacionales [Internet] 2002 [citado 30 jul 2008]; 2(2). Disponible en http://www.facso.uchile.cl

12. Restrepo A. Factores relacionados con la deserción estudiantil en el programa de Enfermería de la Universidad Libre de 
Pereira. Cultura del Cuidado Enfermería. Perú. Anales de la facultad de Medici2010; 7(2): 5-14. na Universidad Nacional Mayor de San

13. Sanabria H. Deserción en estudiantes de Marcos. 2002; 63(4): 301-311. Enfermería en cuatro universidades del 